\title{
Assessment of oral health literacy of principal caregivers and its association with dental caries in Mexican disabled pediatric patients
}

\author{
(D) Julio Carlos Garnica-Palazuelos ${ }^{1}$, (1) Mercedes Bermúdez², (1) Juan Luis Cota-Quintero ${ }^{3}$, (1) Gerardo \\ Bueno-Acuña ${ }^{4}$, (D) Sandra Santana-Delgado ${ }^{5}$, (1) Ana Larrinua-Pacheco ${ }^{6}$, (D) Claudia Bujanda-Ríos ${ }^{7}$, \\ (D) Maricela Ramírez-Álvarez ${ }^{8}$, (D) Carlos Villegas-Mercado ${ }^{9}{ }^{-}$
}

\section{Highlights}

Disabled persons have a higher incidence of caries and periodontal disease than the general population.
Oral health literacy level of principal caregivers was significantly associated with disabled pediatric patient's high result in the DMFT index.
Low oral health literacy level of principal caregivers is a risk factor to have a high DMFT in disabled pediatric patients.
1 Professor, Faculty of Dentistry,

Autonomous University of Sinaloa, Mexico

2 Professor, Faculty of Dentistry,

Autonomous University of Chibuabua,

México

3 Professor, Faculty of Dentistry,

Autonomous University of Sinaloa, Mexico

${ }^{4}$ Professor, Faculty of Dentistry,

Autonomous University of Chibuahua,

México

${ }^{5}$ Professor, Faculty of Dentistry, Autonomous

University of Chibuabua, México

${ }^{6}$ Professor, Faculty of Dentistry, Autonomous

University of Chibuabua, México

7 Professor, Faculty of Dentistry, Autonomous

University of Chibuahua, México

${ }^{8}$ Professor, Faculty of Dentistry, Autonomous

University of Sinaloa, Mexico

${ }^{9}$ Professor, Faculty of Dentistry, Autonomous

University of Chibuabua, México

\section{Correspondence:}

Faculty of Dentistry, Autonomous University of Chihuahua, México Email address:

cmercado@,uach.mx

\section{Abstract}

Aim: To assess oral health literacy (OHL) of principal caregivers (PC) and its association with dental caries in Mexican disabled pediatric patients (DPP). Methods: 40 disabled patients and their PC were recruited from the pediatric dentistry Clinic (UAS) and the Center of Rehabilitation and Special Education of the Institute for Integral Development of the Family in Sinaloa, Mexico. Spanish Oral Health Literacy Scale (SOHLS) was used for the OHL assessment of PC. DPP were subject to clinical examinations to observe the mean number of decayed, missing, or filled teeth through the DMFT index. Disabilities were classified in neuromotor, auditive and language, visual, intellectual, or multiple. To estimate OHL differences, the variable was divided into 3 categories according to the obtained score: low (0-16), medium (17-22), or high (23-29). For the logistic regression model, the variable was categorized on 2 according to ANOVA results: lower (0-22) and high (23-29) to estimate the association between PC low OHL and DMFT percentual increase. Results: Regarding DPP, there were no differences in age expressed in years $(12.47( \pm 8.16)(\mathrm{p}=0.673)), \mathrm{DMTF}(0.34( \pm 0.26)(\mathrm{p}=0.673))$ or the number of teeth $(23.32( \pm 5.29)(\mathrm{p}=0.653)$. Besides, no differences were found in PC age expressed in years $(41.22( \pm 8.91)(\mathrm{p}=0.795))$, PC scholarship expressed in years (3.95( \pm 8.91$)(\mathrm{p}=0.128))$ or PC OHL $(22.27( \pm 3.99)(\mathrm{p}=0.205))$. The logistic regression model categorizing $\mathrm{OHL}$ on high $(>22)$ and low $(\leq 22)$ found $0.97 \%$ more risk to be a PC with low OHL per percentual unit of DMFT increment $(p=0.041)$. Conclusions: The OHL level of PC is significantly associated with DPP's high result in the DMFT.

Keywords: Caregivers; Dental Caries; Dentistry; Literacy

Received: 18 Nov 2021

Accepted: 20 Dec 2021

Online First: 21 Dec 2021 


\section{INTRODUCTION}

According to World Health Organization (WHO), about $15 \%$ of the world population lives with some form of disability, and $2-4 \%$ of them can experience significant difficulties in self-care functions given by developmental or sensitive disorder ${ }^{1,2}$, which result in communicative or intellectual impairments ${ }^{3}$ that conducts to a longterm dependence. ${ }^{4}$ In this regard, critical aspects such as oral health care depend on the knowledge, attitude, and actions of the PC.5,6

This is relevant since oral health is a major constituent of general health. ${ }^{7}$ It is widely reported that disabled persons (DP) have a higher incidence of caries and periodontal disease than the general population. ${ }^{8-11}$ Oral health problems like caries and periodontitis can lead to pain ${ }^{12}$ and eating difficulties, poor nutrition ${ }^{13,14}$, digestive and respiratory problem associated to recurrent infections ${ }^{15,16}$, sleep disturbance ${ }^{17}$, and decreased self-esteem ${ }^{5}$ (Figure 1). Furthermore, dental caries and gingivitis in DP (2-19 years old) have been strongly associated with the level of the mother's education. ${ }^{18}$
Oral health literacy (OHL) plays a central role in the relationship between the perception about signs and symptoms, perceived value of good oral health, and the ability to understand the health care system. ${ }^{19}$ This relationship has been associated with several oral health actions and outcomes. There is evidence that OHL of PC is a strong predictor of oral health in the DP under their care. ${ }^{20}$ Health literacy includes the ability to understand instructions on prescriptions or basic health information, medical education brochures, and health personnel directions. ${ }^{21}$ PC are in charge of preventive oral care, diet, oral hygiene, and attendance at dental consultations. ${ }^{22}$ Besides, there is a strong relation between OHL of PC and decayed, missing, and filled teeth (measured by DMFT index) in the persons under their care. ${ }^{23}$ This is relevant since dental caries is the most incidental disease of the oral cavity in Mexico and worldwide. ${ }^{24}$ Therefore, the purpose of this study was to associate the OHL of PC with dental caries in Mexican DPP.

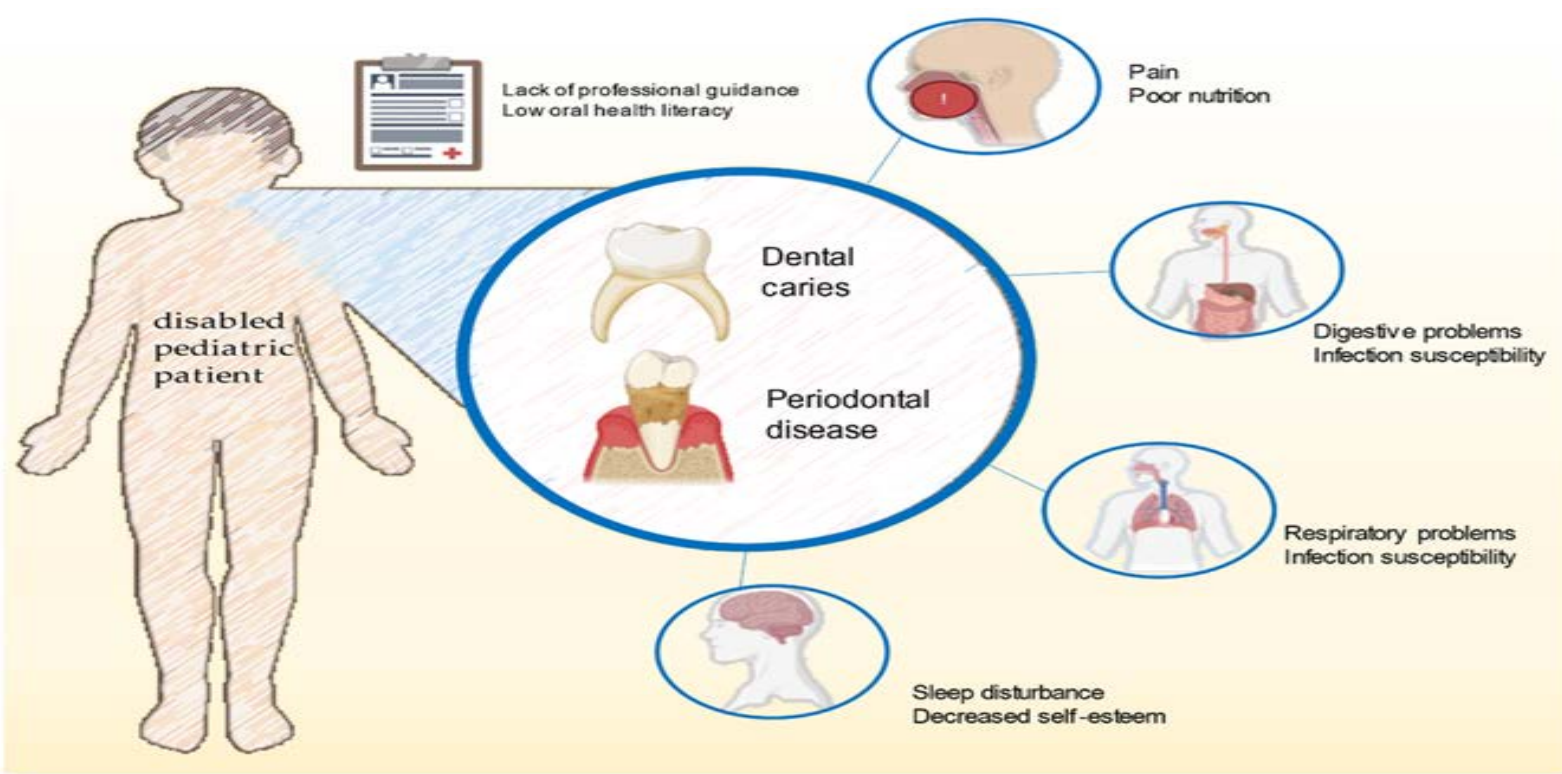

Figure 1. Oral health problems in DPP like caries and periodontitis can lead to other affections such as pain and eating difficulties, poor nutrition digestive and respiratory problem associated to recurrent infections, sleep disturbance, and decreased self-esteem. Lack of professional guidance or low OHL of PC could be key for the development of illness 


\section{METHODS}

\section{Study sample}

A sample size of 8 per disability class was calculated for detecting differences between 2 proportions on DMFT index, estimated by a $\mathrm{X}^{2}$ test, using epidemiologic data of Down's syndrome patients ${ }^{25}$ that reports $37 \%$ on DMFT index vs $77 \%$ of cerebral palsy patients ${ }^{26}$, using a $\alpha=0.05$ and $\beta=0.8$ for a 2 tiled test. In this cross-sectional study, 40 disabled patients and their PC were recruited from the pediatric dentistry Clinic (Universidad Autónoma de Sinaloa UAS) and the Center of Rehabilitation and Special Education of the Institute for Integral Development of the Family in Sinaloa, Mexico. Informed consent was obtained from all subjects involved in the study. This study was previously approved for ethical committee of Universidad Autónoma de Sinaloa. DPP included had more than 20 teeth and their PC accepted to participate and filled the instrument. DPP with genetic syndromes, systemic health problems, illiterate PC, or incomplete questionnaires were excluded.

\section{Measurements}

Spanish Oral Health Literacy Scale SOHLS (reliable and validated instrument ${ }^{27}$ was used for the OHL assessment of PC. This instrument is a self-answered questionnaire for Mexican Spanish speakers that evaluates how a person understands, processes, and applies specific information. It contains 29 items that assess five literacy skills: location, integration, generation, calculation, and return (9 correspond to cycling skills, 4 to location, 2 to formulation, 3 to generation, and 11 to integration skills). Each item has a value of one if answered correctly. The complete test has a summative score from 0 (lowest literacy) to 29 (highest literacy).

\section{DMFT}

DPP were subject to clinical examinations through DMFT Index. This index is the most important used in epidemiological studies of the health status of the community. ${ }^{28}$ The DMFT score of the samples was determined based on the results of clinical examination and calculation of the number of decayed (D), filled (F), and missed (M) teeth due to caries. The data were collected through observation and direct examination using a mirror number \#4. During the inspection, the subjects under examination and the researcher sat close to the window to examine under the maximum natural light.

\section{Disability classification}

Disability classification was analyzed as a confusing variable and decoded in agreement to the Social Integration Law of Sinaloa for disabled people (neuromotor, auditive and language, visual, and intellectual or multiple).

\section{Standardization}

SOHLS standardization was performed with a pilot probe of 10 PC. Complications on lecture, understanding, or tiredness were evaluated during questionnaire filling without finding any problem. DMFT index standardization was performed using intraoral pictures of 10 patients. DMFT results of every patient were assessed by 2 evaluators, and exercises were repeated 3 times in 1 -week intervals using the Intraclass concordance coefficient. A $0.998 \%$ concordance was obtained between evaluators.

\section{Statistical analysis}

Significance and differences were assessed across disability classes using different statistical tests. ANOVA was used for parametric variables. To estimate OHL differences, the variable was divided into 3 categories according to the obtained score: low (0-16), medium (17-22), or 
high (23-29). For the logistic regression model, the variable was categorized on 2 according to ANOVA results: lower (0-22) and high (23-29) to estimate the association between PC low OHL and DMFT percentual increase.

\section{RESULTS}

Descriptive statistic was developed to find differences between disability classes (Table 1). Regarding DPP, there were no differences in age expressed in years $(12.47( \pm 8.16) \quad(\mathrm{p}=0.673))$, DMTF $(0.34( \pm 0.26)(\mathrm{p}=0.673))$ or the number of teeth $\quad(23.32 \quad( \pm 5.29) \quad(\mathrm{p}=0.653) \quad$ showing homogeneity of the main confounding variables of the DPP.
Besides, no differences were found in PC age expressed in years $(41.22( \pm 8.91)(\mathrm{p}=0.795))$, and PC scholarship expressed in years $(3.95( \pm 8.91)$ $(\mathrm{p}=0.128))$ or PC OHL $(22.27( \pm 3.99)(\mathrm{p}=0.205))$.

DMFT analyses associated with PC OHL raised lower values of DMTF related to high OHL group $(0.26( \pm 0.21))$ when compared to medium OHL $(0.42( \pm 0.29))$ or low OHL $(( \pm 0.32) \quad(\mathrm{p}=0.041))$ groups. Logistic regression model categorizing OHL on High $(>22)$ and Low $(\leq 22)$ found $0.97 \%$ more risk to be a PC with low OHL per percentual unit of DMFT increment $(\mathrm{p}=0.041)$ (Table 2). Considering the uniformity of the confounding variables, we assume that the differences detected were not influenced by the main confounding variables.

Table 1. Descriptive analysis for disability class

\begin{tabular}{lllllll}
\hline Disability class & DPP Age & DMFT & Teeth & PC age & $\begin{array}{l}\text { PC } \\
\text { scholarship }\end{array}$ & OHL \\
& & & & & & \\
\hline Neuromotor & $11.40( \pm 6.71)$ & $0.32( \pm 0.3)$ & $23.50( \pm 6.34)$ & $39.90( \pm 7.82)$ & $4.50( \pm 7.82)$ & $23.2( \pm 3.11)$ \\
Auditivea nd & $11.80( \pm 6.80)$ & $0.39( \pm 0.22)$ & $24.30( \pm 3.40)$ & $40.80( \pm 6.17)$ & $4.10( \pm 6.17)$ & $20.9( \pm 5.62)$ \\
language & & & & & & \\
Visual & $15.30( \pm 12.72)$ & $0.37( \pm 0.33)$ & $21.50( \pm 7.33)$ & $43.70( \pm 11.46)$ & $4.60( \pm 11.46)$ & $24.0( \pm 3.09)$ \\
Intellectual or & $11.40( \pm 4.83)$ & $0.29( \pm 0.19)$ & $24.00( \pm 3.23)$ & $40.50( \pm 10.18)$ & $2.60( \pm 10.18)$ & $21.0( \pm 3.16)$ \\
multiple & & & & & & \\
Total & $12.47( \pm 8.16)$ & $0.34( \pm 0.26)$ & $23.32( \pm 5.29)$ & $41.22( \pm 8.91)$ & $3.95( \pm 8.91)$ & $22.27( \pm 3.99)$ \\
\hline
\end{tabular}

DPP: Disabled pediatric patients; DMTF: Index to measure Decay, Missing or filled teeth; PC: Principal Caregivers; OHL:Oral Health Literacy

Table 2. Logistic regression analysis found more risk to be a PC with low OHL per percentual unit of DMTF increment

\begin{tabular}{|c|c|c|c|c|c|c|}
\hline OHL & $\mathbf{N}$ & DMFT & & CI (95\%) & \multicolumn{2}{|c|}{ Significance } \\
\hline Low (10-16) & 4 & $0.54( \pm 0.32)$ & & $0.29-1.05$ & & \\
\hline Medium (17-22) & 15 & $0.42( \pm 0.29)$ & & $0.26-0.57$ & $\mathrm{p}=0.041$ & \\
\hline High (23-28) & 21 & $0.26( \pm 0.21)$ & & $0.17-0.36$ & & \\
\hline Total & 40 & $0.35( \pm 0.26)$ & & $0.26-0.43$ & & \\
\hline Logistic regression & $\mathbf{N}$ & DMFT \% & $\mathbf{X}^{2}$ & CI (95\%) & $\operatorname{Exp}(B)$ & Significance \\
\hline High & 19 & $44( \pm 29)$ & $\mathrm{p}=0.019$ & $30-58$ & 0.970 & $\mathrm{p}=0.041$ \\
\hline
\end{tabular}

OHL: Oral Health Literacy; DMTF: Index to measure Decay, Missing or filled teeth; CI: confidence interval; $\mathrm{X}^{2}$ : squared Chi; Exp (B): exponentiation of the B coefficient. 


\section{DISCUSSION}

The results demonstrate that the OHL level of PC is significantly associated with DPP's high result in the DMFT index. Besides, improving the OHL of PC is the best option to help strengthen their abilities to promote oral health and DPPs general health. These findings are opposite to those obtained by Divaris and Cols in 2012 29 , who found poor correlations between the assessment of PC clinical needs of patients. This difference could be explained given the age of children included in the study who were less than two years old. Besides, as mentioned by Baskaradoss $\&$ Cols in $2018^{20}$, parents of very young children may overestimate the oral health status of their child.

This study has some limitations regarding the use of the DMFT index for dental caries detection, which fails to detect interproximal and incipient lesions. Besides, the DMF score is a count that does not indicate the number of teeth that are at risk and does not discriminate between the mix of decayed, missing, and filled teeth or surfaces, and whether teeth are lost for reasons other than caries; therefore, the validity of the DMF can be considered as reduced. ${ }^{30}$ Also, a larger or multi-centered study can improve the validity of results. In this regard, more work is required to further explore the different aspects of OHL. On the other hand, the strength of this work is the sampling method employed since aimed to provide a comprehensive picture of the DPP in this country, as patients of the study come from different attention centers of Sinaloa and have different socioeconomic and cultural statuses.

In 2019, 1.2 million of DPP were reported in Mexico. The results of this study suggest that the age of DPP, teeth number, as well as descriptive data of PC, have no differences regardless of disability class. These similarities are given by the presence of physical or communicative barriers at the time of oral health care. This could be due to a lack of experience of professionals in the management of information and the lack of adequate guidance to their PC. ${ }^{31}$

Findings showed that Low PC OHL is a risk factor to have a high DMFT in DPP which agrees with data suggesting that patients with lower values of health literacy had a poor general and oral health status. ${ }^{20}$ Besides, there is evidence that shows that PC with high OHL are more attentive to prevent oral health of the patients they take care. ${ }^{32,33}$ As has been previously reported ${ }^{34}$, knowledge and oral health literacy of PC are important predictors that must be taken into account to reduce social inequalities in health through actions undertaken at the local level in patients with disabilities.

Dental caries in Mexico is a public health problem. Therefore, the promotion of primary prevention and understanding of oral health care in DPP should be an integral part of dentistry. These individuals are a risk group ${ }^{35}$ since from a young age they require specialized dental care. Besides, it is important to ensure PCs adequate literacy given by trained professionals that allows understanding preventive care, the importance of diet, frequency of visit to the dentist, and other instructions to guarantee oral health of DPP.

\section{CONCLUSIONS}

The OHL level of PC is significantly associated with DPP's high result in the DMFT index. Improving the OHL of PC might, therefore, help strengthen their capacities to promote oral health, thus helping to improve DPP general health.

\section{REFERENCES}

1. World Health Organization. World report on disability [Internet] 2020 [cited 2021 Nov 15]. Available from: 
https://www.who.int/disabilities/world_report/2 011/report.pdf

2. Allerton LA, Welch V, Emerson E. Health inequalities experienced by children and young people with intellectual disabilities: a review of literature from the United Kingdom, J Intellect Disabil 2011;15:269-278

3. Raina P, O'Donnell M, Rosenbaum P, Brehaut J, Walter SD, Russell D, Swinton M et al. The health and well-being of caregivers of children with cerebral palsy, Pediatrics 2015;115:626-636

4. Shonkoff JP, Hauser-Cram P, Krauss MW, Upshur CC. Development of infants with disabilities and their families: implications for theory and service delivery. Monogr Soc Res Child Dev 1992;57:1-153

5. Anders PL, Davis EL. Oral health of patients with intellectual disabilities: A systematic review. Spec Care Dentist 2011;30:110-117

6. Bastawrous M. Caregiver burden--a critical discussion. Int J Nurs Stud 2013;50:431-441

7. Petrovic BB, Peric TO, Markovic, DL, Bajkin BB, Petrovic D, Blagojevic DB, Vujkov S. Unmet oral health needs among persons with intellectual disability. Res Dev Disabil 2016;59: 370-377

8. Leal RL, Vieira de Lima Saintrain M, Pimentel Gomes Fernandes Vieira-Meyer A. Access to dental public services by disabled persons. BMC Oral Health 2015;15: 35-42

9. Jaber MA. Dental caries experience, oral health status and treatment needs of dental patients with autism. J Appl Oral Sci 2011;19:212-217

10. Makkar A, Indushekar KR, Saraf BG, Sardana $\mathrm{D}$, Sheoran N. A cross sectional study to evaluate the oral health status of children with intellectual disabilities in the National Capital Region of India. J Intellect Disabil Res 2019;63:31-39

11. Lee LY, Lim KC, Kim SY, Paik HR, Kim YJ, and Jin BH. Oral health status of the disabled compared with that of the non-disabled in Korea: A propensity score matching analysis. PLoS One 2019;14:0208246

12. Mathur VP, Dhillon JK. Dental Caries: A Disease Which Needs Attention. Indian J Pediatr 2018;85:202-206

13. So M, Ellenikiotis YA, Husby HM, Paz, CL, Seymour B, Sokal-Gutierrez K. Early Childhood Dental Caries, Mouth Pain, and Malnutrition in the Ecuadorian Amazon Region. Int J Environ Res Public Health 2017;14:1-12

14. Gondivkar SM, Gadbail, AR, Gondivkar, RS, Sarode SC, Sarode GS, Patil S, Awan KH.

Nutrition and oral health. Dis Mon 2019;65:147154

15. Grund K, Goddon I, Schüler IM, Lehmann T, Heinrich-Weltzien R. Clinical consequences of untreated dental caries in German 5- and 8-yearolds. BMC Oral Health 2015;15:140-148

16. Mojon P, Budtz-Jørgensen E, Michel JP, Limeback H. Oral health and history of respiratory tract infection in frail institutionalised elders. Gerodontology 1997;14: 9-16

17. Dumitrescu AL, Toma C, Lascu V. Associations among sleep disturbance, vitality, fatigue and oral health. Oral Health Prev Dent 2010;8:323-330

18. Cristina GD, Jorge MS, César BJ, Sotille França BH, Tetu MS. Health promoting schools and their impact on the oral health of mentally disabled people in Brazil. Health Promot Int 2010;25: 425-434

19. Lacy NL, Paulman A, Reuter MD, and Lovejoy B. Why we don't come: patient perceptions on no-shows. Ann Fam Med 2004;2:541-545

20. Baskaradoss JK. Relationship between oral health literacy and oral health status. BMC Oral Health 2018;18:172-180

21. Wehmeyer MM, Corwin CL, Guthmiller JM, Lee JY. The impact of oral health literacy on periodontal health status, J Public Health Dent 2014;74:80-87

22. Phlypo I, Janssens L, Palmers E, Declerck D, Marks L. Review of the dental treatment backlog of people with disabilities in Europe. J Forensic Odontostomatol 2019;3:42-49

23. Bridges SM, Parthasarathy DS, Wong HM, Yiu CK, Au TK, McGrath CP. The relationship between caregiver functional oral health literacy and child oral health status. Patient Educ Couns 2014;94:411-416

24. Vergnes JN, Mazevet M. Oral diseases: a global public health challenge. The Lancet 2020;395:186-192

25. Oliveira AC, Czeresnia D, Paiva SM, Campos MR, Ferreira EF. Utilization of oral health care 
for Down syndrome patients. Rev Saude Publica 2008;42:693-699

26. Giménez Prat MJ, López Jiménez J, Boj Quesada JR. Estudio epidemiológico de la caries en un grupo de niñoscon parálisis cerebral. Medicina Oral S. L 2003;8:45-50

27. Villanueva VMC, Wintergerst A, Borges YSA. Toward a Comprehensive Instrument of Oral Health Literacy in Spanish. J Health Commun 2015;20:930-937

28. Broadbent JM, Thomson WM. For debate: problems with the DMF index pertinent to dental caries data analysis. Community Dent Oral Epidemiol 2005;33:400-409

29. Divaris K, Vann WFJ, Baker AD, Lee JY. Examining the accuracy of caregivers' assessments of young children's oral health status. Journal of the American Dental Association 2012;143:12371247

30. Shulman JD, Cappelli DP. Epidemiology of dental caries. In: Cappelli DP, Mobley CC, editors. Prevention in Clinical Oral Health Care. Mosby: Saint Louis; 2008. p 2-13

31. Giraldo-Zuluaga MC, Martínez-Delgado CM, Cardona-Gómez N, Gutiérrez-Pineda JL, Giraldo-Moncada KA, Jiménez-Ruíz PM. Manejo de la salud bucal en discapacitados. Artículo de revision. CES Odontología 2017;30:23-36 32. Mejia GC, Weintraub JA, Cheng NF, Grossman W, Han PZ, Phipps KR, Gansky SA. Language and literacy relate to lack of children's dental sealant use. Community Dent Oral Epidemiol 2011;39:318-324

33. Yazdani R, Esfahani EN, Kharazifard MJ. Relationship of Oral Health Literacy with Dental Caries and Oral Health Behavior of Children and Their Parents. J Dent (Tehran) 2018;15:275-282 34. Marquillier T, Lombrail P, Azogui-Lévy S. Social inequalities in oral health and early childhood caries: How can they be effectively prevented? A scoping review of disease predictors. Rev Epidemiol Sante Publique 2020;68:201-214

35. American Academy of Pediatric Dentistry. Definition of special health care needs. Pediatr Dent 2018;30:16-16

\section{How to cite this article:}

Julio Carlos Garnica-Palazuelos, Mercedes Bermúdez, Juan Luis Cota-Quintero, Sandra Santana-Delgado, Ana Larrinua-Pacheco, Maricela Ramírez-Álvarez, Carlos Villegas-Mercado. Assessment of oral health literacy of principal caregivers and its association with dental caries in Mexican disabled pediatric patients. Contemp Pediatr Dent 2021:2(3):151-157.

\section{Declarations}

Acknowledgements: Authors acknowledge Dr. Adriana Garza for belping to conceive the design of this study.

Conflict of Interest Statement: Authors disclose no potential conflicts of interest.

Ethics Statement: Informed consent was obtained from all subjects involved in the study. This study was previously approved for ethical committee of Universidad Autónoma de Sinaloa.

Informed Consent: Informed consent were obtained form all participants and legal guardians.

Author contributions: Conception and design: All Authors; Acquisition of data: JCGP, MB, JLCQ CEVM; Interpretation of data: JCGP, MB, CEVM; Drafting article: $M B, G B A, S S D, A L P, C B R, M R A$, CEVM; Revision artice: $M B$, JLCQ, GBA, SSD, ALP, CBR, MRA, CEVM; Final approval: All Authors

Funding: This work is not finantiated.

Data Availability: The data used to support the findings of this study can be made available upon request to the corresponding author.

Peer-review: Externally double-blinded peer-reviewed. 